\title{
L'évolution de la jurisprudence espagnole sur la qualification des coursiers employés par les plateformes
}

\section{Tania Bazzani}

Traducteur : Sylvain Giovanni Nadalet

\section{(2) OpenEdition}

\section{Journals}

Édition électronique

URL : https://journals.openedition.org/rdctss/746

DOI : $10.4000 /$ rdctss. 746

ISSN : 2262-9815

\section{Éditeur}

Centre de droit comparé du travail et de la sécurité sociale

\section{Édition imprimée}

Date de publication : 1 avril 2021

Pagination : 176-181

ISSN : 2117-4350

\section{Référence électronique}

Tania Bazzani, «L'évolution de la jurisprudence espagnole sur la qualification des coursiers employés par les plateformes », Revue de droit comparé du travail et de la sécurité sociale [En ligne], 1| 2021, mis en ligne le 01 avril 2021, consulté le 01 avril 2022. URL : http://journals.openedition.org/rdctss/746 ; DOI : https://doi.org/10.4000/rdctss.746

Revue de droit comparé du travail et de la sécurité sociale est mise à disposition selon les termes de la Licence Creative Commons Attribution - Pas d'Utilisation Commerciale - Pas de Modification 4.0 International. 


\title{
TANIA BAZZANI
}

\author{
UNIVERSITÉ EUROPÉENNE VIADRINA FRANCFORT (ODER)
}

\section{L'ÉVOLUTION DE LA JURISPRUDENCE ESPAGNOLE SUR LA QUALIFICATION DES COURSIERS EMPLOYÉS PAR LES PLATEFORMES*}

La question du statut juridique des coursiers travaillant pour les plateformes de livraison a été dès 2018 débattue dans la jurisprudence espagnole qui les a qualifiés parfois de travailleurs autonomes économiquement dépendants («TRADE»), parfois de travailleurs subordonnés ${ }^{1}$. Ce n'est que dans une phase ultérieure et finale, après qu'une trentaine de décisions aient été rendues à différents niveaux, qu'il a été possible suite à une décision de Cour suprême du 25 septembre $2020^{2}$, de consolider l'orientation favorable à la subordination qui s'était progressivement affirmée devant les juges du fond. Après une présentation des orientations suivies par la jurisprudence espagnole et des principaux critères de subordination juridique utilisés par les juges du fond (I), cette contribution commentera la décision de la Cour du Suprême qui a tranché la question en reprenant certains des critères de la subordination utilisés par les juges du fond (II).

\section{LES CRITÈRES DE LA SUBORDINATION JURIDIQUE UTILISÉS PAR LES JUGES DU FOND DANS LES AFFAIRES CONCERNANT LES COURSIERS À VÉLO}

L'application restrictive des critères de la subordination par les juges du fond (A) a conduit dans un premier temps à une approche des contrats liant les coursiers aux plateformes favorable à l'autonomie (B), jusqu'à ce que d'autres juges du fonds ne renversent cette orientation $\mathbf{( C )}$.

\section{A - LES CRITÈRES DE LA SUBORDINATION JURIDIQUE}

De manière générale, tous les jugements qui s'expriment sur la nature salariée ou non du rapport de travail entre le coursier et la plateforme vérifient si, dans le cas d'espèce, on trouve les éléments typiques de la subordination dégagés par la jurisprudence. L'article 1.1 du Statut du travailleur espagnol (ci-après «ET») ne contient pas de définition du contrat de travail, mais établit les caractéristiques générales permettant de le distinguer des autres types de contrat ${ }^{3}$. II existe à cet égard une présomption de travail salarié lorsqu'un

* Traduction de Sylvain Giovanni Nadalet, Université de Vérone.

1 Voir par exemple F. Pérez de los Cobos Orihuel (Cur.), El trabajo en plataformas digitales. Análisis de su situación jurídica y regulación futura, W. Kluwer, 2018 ; M. Rodríguez-Piñero Royo, M. Hernández Bejarano (Cur.), Economía colaborativa y trabajo en plataforma: realidades y desafíos, Bomarzo, 2017; A. Todolí Signes, A. Todolí Signes, El trabajo en la era de la economía colaborativa, Tirant lo Blanch, 2017.

2 Tribunal Supremo, Sentencia n̊805/2020 du 25 septembre.

3 Sentencia Tribunal Supremo (STS) 03/11/05-rec. 2109 / 04 - EDJ 2005/55243. Art. 1.1 ET : « La présente loi s'applique aux travailleurs qui fournissent volontairement leurs services rémunérés pour le compte d'autrui, et dans le cadre de l'organisation et de la gestion de l'activité par une personne, physique ou morale, appelée employeur ou entrepreneur ». 
rapport juridique est établi entre ceux qui fournissent un service rémunéré et ceux qui en bénéficient (art. $8 \mathrm{ET}$ ).

Le juge est tenu de prêter attention aux circonstances de chaque cas d'espèce, quelle que soit la qualification donnée par les parties au contrat ${ }^{4}$, en vérifiant s'il existe dans les faits la présence d'éléments tels que la rémunération, le travail pour autrui (ajenidad) et la dépendance, qui ont été définis par la jurisprudence pour déterminer si on est en présence d'un contrat de travail.

Ainsi, la situation de dépendance, qui implique une soumission du salarié à la sphère du pouvoir de l'employeur, doit être appréhendée de manière flexible et non rigide. Le travail pour le compte d'autrui consiste en revanche à accepter par anticipation le transfert des fruits du travail au bénéfice de l'employeur qui s'engage à son tour à verser une rémunération au travailleur et dans le fait qu'il revient seulement à l'employeur de prendre et d'assumer les risques économiques de l'activité 5 .

Il est par conséquent essentiel de rechercher la présence ou non de ces caractéristiques typiques de la subordination juridique dans la relation établie entre le coursier et la plateforme.

\section{B - UNE JURISPRUDENCE FAVORABLE AU CARACTÈRE AUTONOME DE LA PRESTATION}

La lecture de la jurisprudence espagnole en faveur de l'autonomie des coursiers ${ }^{6}$ montre que celle-ci portait son attention sur l'absence de pouvoir disciplinaire de l'entreprise et sur la capacité du coursier à s'auto-organiser. Elle mettait notamment l'accent sur le fait que la société ne pouvait pas appliquer de sanction en cas de non-acceptation de commandes même en cas d'abandon d'une commande préalablement acceptée. Elle mettait en exergue également la liberté pour le coursier de choisir la plage horaire durant laquelle il souhaitait travailler et la possibilité pour lui de modifier ses disponibilités, sans être sanctionné à partir du moment où il présentait une justification. Dans ces décisions, la géolocalisation via le GPS n'était pas considérée comme un outil de contrôle de l'entreprise, mais comme un moyen de relever le kilométrage et de déterminer le montant de la rémunération à verser au coursier, le seul but de l'application étant de minimiser les coûts. En outre, le livreur n'avait pas à justifier ses absences, mais seulement à les communiquer à l'entreprise, de sorte qu'il pouvait donc librement décider de ses jours de congé. D'autres éléments étaient aussi mis en avant par la doctrine favorable à l'autonomie du travailleur : l'exécution personnelle de la livraison; une affiliation à la sécurité sociale via le régime spécial des travailleurs indépendants; une rémunération à la tâche et non au temps et l'engagement possible de la responsabilité du coursier concernant la bonne exécution de sa prestation. Enfin, en l'absence de pacte d'exclusivité entre les parties et le livreur, celui-ci pouvait simultanément fournir des services à d'autres entreprises tant que les paramètres de dépendance économique étaient respectés pour conserver le statut du travailleur autonome économiquement dépendant (TRADE, voir ci-après §lI).

Il ressort de cette jurisprudence que l'interprétation donnée par les juges sous-estimait souvent la façon dont la structure très particulière de la plateforme, grâce aux nouvelles

4 SSTS 17 / 06/10 -rcud 3847/09 - EDJ 2010/140235; e 03/05/11 -rcud 2228/10 - EDJ 2011/120826.

5 JS de Madrid Sentencia n53-2019 du 11 février.

6 En 2018: JS n³9 de Madrid, Sentencia (Sent.) n²84/2018 du 3 septembre. En 2019: JS Madrid, n¹7, Sent. n¹2/2019 du 11 janvier; JS Valencia (C. Valenciana), n5, Sent. n¹97/2019 du 10 juin. En 2019, second degré de juridiction: TSJ de Madrid, Sent. $n^{\circ} 715 / 2019$ du 19 septembre. 
technologies, conditionne l'organisation du travail, ce qui a été peu à peu pris en compte par une jurisprudence contraire.

\section{C - UNE JURISPRUDENCE FAVORABLE À LA SUBORDINATION JURIDIQUE}

Les décisions qui ont qualifié de contrat de travail les rapports juridiques existants entre les coursiers et les entreprises propriétaires des plateformes, ont une approche diamétralement opposée ${ }^{7}$. Pour les juges participant de ce courant jurisprudentiel, l'entreprise organise l'activité des coursiers via l'application que le travailleur est obligé de télécharger sur son téléphone. S'agissant de la soumission au pouvoir disciplinaire et de direction de l'employeur, ils observent que tout en pouvant choisir les plages horaires de travail, le travailleur ne peut le faire que dans l'éventail des possibilités préalablement établies par l'entreprise. Enfin l'entreprise donne des consignes au livreur fixant la durée des courses, les règles de comportement à respecter et peut géolocaliser le travailleur à tout moment, ainsi que le sanctionner au cas où il refuserait le service proposé. On mettra l'accent ici sur deux aspects particulièrement importants relevés par les juges : le premier porte sur les caractéristiques du travail pour le compte d'autrui et sur la dépendance, le second sur le caractère non exclusif de la prestation.

S'agissant de la question de l'interprétation des éléments de la subordination dans le contexte du recours à des outils technologiques, on citera le jugement du Tribunal Social de Madrid (Sentencia n53-2019) du 11 février 2019, qui fut la première décision à prendre en compte, à des fins de la qualification juridique, l'impact de l'utilisation des plateformes. Le tribunal constata en particulier une atomisation des heures de travail liée à la fragmentation de l'activité des coursiers en "micro-tâches » faisant en sorte que les périodes de temps libérées n'étaient en réalité pas vraiment utilisables par les travailleurs. Dans ce contexte, la faculté de décider des jours et des heures de travail, comme celle d'accepter des prestations spécifiques, n'a de fait aucune répercussion sur le bon fonctionnement de l'entreprise, étant donné que celle-ci dispose d'un si large éventail de coursiers disposés à travailler que l'absence de certains d'entre eux est automatiquement palliée par les services fournis par d'autres, même lorsqu'un coursier refuse d'effectuer une prestation déjà assignée. Le juge observa de surcroît dans cette décision que, si un coursier décidait d'entreprendre ce type d'activité en tant qu'indépendant, cette activité serait vouée à l'échec. Ses chances en tant qu'entrepreneur seraient pratiquement nulles, puisque le succès de ce type de plateforme est dû au support technique fourni par l'application et à la puissance de sa marque, éléments qui confirment l'impossibilité d'accéder au marché sans ce type d'appuis.

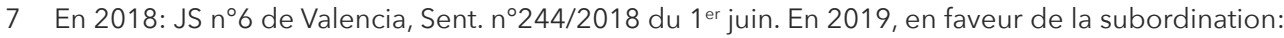
JS n³3 de Madrid, Sent. n53/2019 du 11 février ; JS n¹ de Gijón, Sent. n61/2019 du 20 février ; JS n¹ de Madrid Sent. n¹28/2019 du 3 avril; JS n¹ de Madrid, Sent. n¹34/2019 du 4 avril ; JS n³1 de Barcelona, Sent. n¹93/2019 du 11 juin; JS n³ de Oviedo, Sent. n³29/2019 du 5 juillet. Second degré de juridiction: le Tribunal Superior (TSJ): TSJ de Madrid, Sent. n¹223/2019 du 18 décembre; TSJ de Madrid, Sent. n¹155/2019 du 27 novembre; TSJ de Asturias, Sent. n¹818/2019 du 25 juillet. En 2020: JS n² de Zaragoza, Sent. n¹23/2020 du 27 avril ; JS n²1 de Madrid, Sent. n¹19/2020 du 11 juin; JS n²1 de Barcelona, Sentencia du 7 septembre 2020 ; JS n²4 de Barcelona, Sent. n²59/2020 du 18 novembre ; JS n 4 de Santander, Sent. n²89/2020 du 20 novembre. Second degré de juridiction: le Tribunal Superior (TSJ), en 2020, a statué en faveur de la subordination; TSJ de Cataluña, Sent. n¹449/2020 du 12 mai ; TSJ de Cataluña, Sent. n¹432/2020 du 7 mai ; TSJ de Castilla y León, Valladolid, Sentencia n²91/2020 du 17 février ; TSJ de Madrid, Sent. n68/2020 du 3 février ; TSJ de Cataluña, Sent. n²405/2020 du 11 juin ; TSJ de Cataluña, Sent. n²557/2020 du 16 juin ; TSJ de Galicia, Sent. du 18 novembre 2020 ; TSJ a de Cataluña, Sent. n4021/2020 du 22 septembre. 
Cette approche des juges du fond rejoint celle de la Cour Suprême, qui admet que les critères de la dépendance-subordination ont pu évoluer depuis la création du droit du travail. Une décision de la Cour suprême du 11 mai 1979 avait déjà nuancé la notion de subordination, considérant que la dépendance n'impliquait pas une subordination absolue, mais seulement une intégration dans le cadre organisationnel et disciplinaire de l'entreprise. Dans la société post-industrielle, la notion de dépendance est naturellement devenue plus flexible. Les innovations technologiques sont à l'origine de systèmes de contrôle numérisés pour la fourniture de services. Ces nouvelles formes d'organisation obligent à adapter les notions de dépendance et de salariat ${ }^{8}$. Sur le plan de la méthode, en raison des difficultés à apprécier dans certains cas la présence des éléments typiques et déterminants de la relation de travail subordonnée, il convient d'adopter une technique prenant en compte les circonstances du cas d'espèce afin de voir si elles concordent et permettent, au cas par cas, de retrouver les les indices de l'existence d'un contrat de travail.

S'agissant du critère de l'absence d'exclusivité, qui est déterminant pour la qualification de travailleur autonome, un récent arrêt de la Cour catalane du 7 septembre 2020 a retenu une argumentation qui sera reprise par la Cour suprême dans son arrêt du septembre 2020, analysé ci-après. Cet arrêt présente un intérêt particulier car il va à contre-courant de l'arrêt de la CJUE d'avril 2020, lequel avait retenu une lecture du travail des coursiers sous l'angle de l'autonomie en raison de la possibilité pour le livreur d'être remplacé, de sous-traiter son activité et d'offrir en même temps ses services à des tiers (sans que le juge européen ne s'interroge d'ailleurs sur le point de savoir si le remplacement ou la fourniture à un tiers étaient concrètement possibles). L'arrêt catalan donne une appréciation différente de ces éléments (substitution et non-exclusivité), qui ne seraient pas si déterminants pour retenir le caractère subordonné ou non de la relation de travail au regard du droit national. II observe, en effet que selon la législation espagnole, le prestataire de services est considéré comme un travailleur même s'il n'y a pas de régime d'exclusivité pour l'entreprise, et dans certains cas, même en cas de remplacement par d'autres travailleurs.

\section{II - L'ARRÊT DE LA COUR SUPRÊME DU 25 SEPTEMBRE 20209}

La question centrale de cet arrêt porte sur la détermination du caractère subordonné ou non de la relation établie entre le coursier et le propriétaire de l'application, en l'occurrence Glovo. Dans cette affaire, un coursier s'était pourvu en cassation contre l'arrêt du Tribunal Superior de Justicia de Madrid du 19 septembre 2019, qui s'était exprimé en faveur de la nature autonome du rapport contractuel (confirmant ainsi un jugement de premier ressort). Le coursier entendait s'appuyer sur la jurisprudence qui, dans une affaire similaire, était en faveur de la nature subordonnée du travail. Le pourvoi avait donc pour but de demander à la Cour suprême d'unifier la jurisprudence en la matière. II convient de rappeler les circonstances de l'affaire (A), avant d'observer la façon dont la Cour constate la présence des éléments de dépendance (B) et de travail pour autrui (salariat) (C).

\section{A - LE CADRE CONTRACTUEL}

Sur le plan des rapports juridiques, Glovo, propriétaire d'une plate-forme informatique qu'elle utilise pour ses activités, conclut une série d'accords avec des entreprises locales qui proposent des produits et services. En utilisant la plateforme Glovo, le consommateur final peut acheter ces produits via un mandat donné à la plateforme, qui facture le coût

8 UGT, Servicio de Estudios de la Confederación, Comentario a la STS 805/2020, du 25 Septembre 2020. Caso « Riders ». Un Final Necesario, 13.10.2020.

9 Tribunal Supremo, Sentencia n805/2020 du 25 septembre. 
du produit et du transport. Glovo propose à un coursier de se rendre chez le client local sous contrat, de récupérer le produit et de l'acheminer au consommateur final. Dans le cas d'espèce, les deux parties, le coursier et Glovo, avaient signé un contrat commercial prévoyant une prestation de services professionnelle consistant dans l'exécution de commandes ou de micro-tâches. À raison de ce contrat, le livreur s'était inscrit au régime spécial des travailleurs indépendants. L'activité entre le livreur et Glovo était gérée via l'application de l'entreprise. Le demandeur indiquait à Glovo le créneau horaire sur lequel il était disponible et souhaitait travailler, en s'identifiant au moyen de son téléphone portable: à partir de ce moment, les commandes (slots) étaient insérées en fonction de ce créneau horaire et de sa zone géographique ; l'attribution des commandes avait lieu selon le système d'allocation automatique géré électroniquement par l'algorithme Glovo.

Le contrat commercial en cause stipulait la qualification juridique du travailleur comme TRADE, c'est-à-dire comme travailleur autonome économiquement dépendant, conformément à la loi espagnole (RCL 2007, 1354).

Cependant, pour acquérir ce statut, certains éléments devaient être réunis:

1- le coursier devait s'organiser de manière autonome pour exercer son activité, même si en réalité, comme l'observera la Cour, celui-ci obéissait au strict respect des règles établies par Glovo ;

2- le coursier devait aussi disposer de sa propre infrastructure et du matériel pour l'exercice de l'activité, indépendamment de celle de son client.

En réalité, le travailleur ne disposait que d'un motocycle et d'un téléphone portable, qui pouvaient être considérés comme des moyens accessoires ou complémentaires. Selon la Cour, en effet, l'infrastructure essentielle pour mener à bien cette activité est le programme informatique développé par Glovo (l'application), lequel met les entreprises en contact avec les clients finaux, de sorte que le livreur ne dispose pas d'une véritable infrastructure qui lui permette d'opérer seul. Glovo fournissait également au livreur une carte de crédit pour pouvoir acheter les produits destinés au client final et compensait financièrement le temps d'attente, c'est-à-dire le temps qu'il passait sur le lieu de prise en charge de la commande. Le contrat commercial reconnaissait au livreur la possibilité de refuser des clients ou des services, de choisir le créneau horaire de ses services ou de fournir un service identique pour d'autres plateformes. En réalité, cette autonomie est jugée plus théorique que réelle, puisque le coursier se trouve dans une situation de dépendance vis-à-vis de Glovo. Le choix du créneau horaire est clairement conditionné par un système de pénalités et de points qui fait en sorte que les coursiers ayant les scores les plus élevés bénéficient d'un accès préférentiel aux commandes. La Cour a précisément concentré son analyse sur l'organisation du travail établie par Glovo pour déceler des situations de dépendance typiques de la subordination juridique qu'elle génère.

\section{B - LES MODES DE DIRECTION ET DE CONTRÔLE DU TRAVAIL DU COURSIER : LE CRITÈRE DE LA DÉPENDANCE}

La Cour a accordé une attention particulière à la question du système de notation et de pénalité auquel le coursier était soumis. Le score dépendait de trois facteurs: l'évaluation du client final, l'efficacité démontrée dans l'exécution des commandes les plus récentes et la performance du service aux heures de pointe. Par ailleurs, il y avait une pénalité de 0,3 point chaque fois qu'un livreur n'opérait pas sur le créneau horaire qu'il avait initialement retenu. Selon la Cour, ce score affecte de fait la liberté de choix des horaires du coursier car s'il n'est pas disponible pour fournir ses services de livraison sur les plages horaires les plus demandées, alors son score diminue et, avec lui, la possibilité de recevoir plus de commandes à l'avenir. La société sanctionnait en outre les coursiers, en cessant de leur fournir des commandes, lorsqu'ils n'opéraient pas sur les créneaux qu'ils avaient retenus, à 
moins d'avoir dûment communiqué un motif justificatif. La conséquence d'un tel système est que les coursiers se disputaient en fait les créneaux horaires les plus rémunérateurs, sans aucune garantie de commande minimale. II s'agissait d'un système qui, en fait, n'exigeait pas de respecter un programme rigide imposé par l'entreprise, car ces micro-tâches étaient réparties sur un nombre tel de coursiers que cela garantissait une main-d'œuvre suffisante pour compenser le refus d'un créneau horaire ou d'un service refusés par un autre livreur. Le coursier était également soumis à l'évaluation finale du client. Or la mise en place de systèmes de contrôle de production basés sur l'évaluation des clients représente pour la Cour un indicateur de la nature subordonnée de la prestation. L'entreprise donnait par conséquent des instructions précises aux coursiers afin de maîtriser le processus de production: le coursier devait effectuer la prestation dans le délai maximum convenu; il lui était interdit de porter des logos d'entreprise tels que des t-shirts, des chapeaux, etc.

Glovo avait ainsi mis en place des moyens de direction et de contrôle ayant un impact direct sur l'activité du livreur et pas seulement sur le résultat, par le biais d'un algorithme. La compensation financière versée par Glovo à ce dernier dépendait des critères établis par l'entreprise elle-même. Le système de contrôle constant auquel le coureur était soumis, par géolocalisation GPS, ne faisait que confirmer l'existence d'un contrat de travail. Enfin, le contrat TRADE établi par la société et signé par les parties comprenait treize motifs possibles de résiliation, centrés sur d'éventuelles violations contractuelles par le livreur, qui coïncidaient avec les motifs classiques d'un licenciement disciplinaire.

\section{C - LES DIFFÉRENTES FORMES DE TRAVAIL POUR AUTRUI}

Plusieurs caractéristiques du rapport contractuel établissant la situation de salariat, précédemment décrite, figuraient dans le cas d'espèce. En premier lieu, l'aliénation des bénéfices résultait du fait que Glovo s'appropriait directement le résultat des prestations de travail, ce qui profitait à cette société. Le coursier n'était pas non plus impliqué dans les accords établis entre Glovo et les commerçants, ni dans les relations entre Glovo et les clients auxquels les commandes étaient servies. Du point de vue des risques, si d'une part le coursier assumait le risque de la bonne exécution de la livraison, répondant de tout dommage envers l'utilisateur final (le client), pesait sur également lui le risque d'accident et les frais d'entretien du motocycle, de sorte que la combinaison risque-profit typique du libre exercice des professions autonomes était ici absente.

Enfin, comme l'avaient observé certains juges du fond, les moyens de production essentiels dans cette activité n'étaient pas le téléphone portable ni le deux-roues utilisé pour les livraisons, mais plutôt la plateforme numérique de Glovo, à laquelle les restaurants, les consommateurs et les livreurs devaient s'inscrire, et sans laquelle la fourniture du service n'aurait pas été possible.

\section{Conclusion}

Par l'arrêt du 25 septembre 2020, le Tribunal suprême espagnol a unifié la jurisprudence en faveur d'une requalification en contrat de travail de la relation de travail entre les coursiers et les plateformes, du fait de la présence des éléments de dépendance, de salariat et des modalités de rémunération retenues. Le coursier est vu comme un travailleur inséré dans l'organisation productive d'autrui, soumis au pouvoir de gestion et de contrôle de l'entreprise. La jurisprudence espagnole démontre ainsi qu'elle a su adopter une approche tendant à adapter les instruments typiques du droit du travail (les critères de la subordination juridique) au nouveau contexte productif de la Gig Economy. 